\title{
VORSCHLÄGE FÜR EFFEKTIVERES FREMDSPRACHENLERNEN AN ÖFFENTLICHEN SCHULEN
}

\author{
Necmettin YAMAN ${ }^{1}$
}

\begin{tabular}{|l|l|}
\hline Anahtar Kelimeler & Zusammenfassung \\
\hline $\begin{array}{l}\text { Fremdsprachenlernen } \\
\text { Lernprobleme }\end{array}$ & $\begin{array}{l}\text { An den staatlichen Schulen der Türkei bekommen die Lernenden 11 Jahre lang } \\
\text { Hunderte von Stunden Fremdsprachenunterricht, merken die meisten Lernenden } \\
\text { jedoch am Ende der Schule, dass sie fast kein Wort von diesen Fremdsprachen } \\
\text { Vorbereitungsklasse } \\
\text { Mëhoden und Techniken } \\
\text { Ziellosigkeit, die falschen Lehrmethoden, der Bedarf an gut ausgebildete Lehrkräfte } \\
\text { und die unvernünftige Zeitplanung die wichtigsten zu sein scheinen. Ziel dieser }\end{array}$ \\
\hline Makale Bilgisi & $\begin{array}{l}\text { Forschung ist es, einen effektiveren Fremdsprachenunterricht an öffentlichen Schulen } \\
\text { zu ermöglichen. Es wird erwartet, dass Fremdsprachenlernen an den öffentlichen } \\
\text { Schulen produktiver wird, wenn die vorgesehenen Vorschläge umgesetzt werden } \\
\text { können. Die durch Literaturrecherchen festgestellten Probleme beim }\end{array}$ \\
\hline Araştırma Makalesi & $\begin{array}{l}\text { Fremdsprachenlernen an den öffentlichen Schulen und die dafür vorgeschlagenen } \\
\text { Lösungen wurden den Lehrern, die die meisten Erfahrungen zu diesem Thema } \\
\text { haben, mittels eines Fünf-Punkte-Likert-Fragebogens zu ihren Meinungen präsentiert. } \\
\text { Diese festgestellten Probleme und deren Lösungsvorschläge wurden auch durch die } \\
\text { Umfrage unterstützt. }\end{array}$ \\
\hline $\begin{array}{l}\text { Gönderim Tarihi: } 07.10 .2021 \\
\text { Kabul Tarihi: 26.11.2021 } \\
\text { Yayın Tarihi: } 08.12 .2021\end{array}$ &
\end{tabular}

\section{SUGGESTIONS FOR MORE EFFECTIVE FOREIGN LANGUAGE LEARNING IN PUBLIC SCHOOLS}

\begin{tabular}{|c|c|}
\hline Keywords & Abstract \\
\hline $\begin{array}{l}\text { Foreign language learning } \\
\text { Learning problems } \\
\text { Proposed solutions } \\
\text { Preparatory class } \\
\text { Methods and techniques }\end{array}$ & $\begin{array}{l}\text { Students in public schools in Turkey receive hundreds of hours of foreign language } \\
\text { lessons for } 11 \text { years, however most of them realize at the end of school that they can } \\
\text { hardly speak a word of these foreign languages. There are many causes of which lack } \\
\text { of motivation, aimlessness, incorrect teaching methods, need for well-trained } \\
\text { teachers, and unreasonable limited hours seem to be the most important. The aim of } \\
\text { this research is to enable more effective foreign language teaching in public schools. }\end{array}$ \\
\hline Article Info & Foreign language learning in public schools is expected to be more productive if the \\
\hline 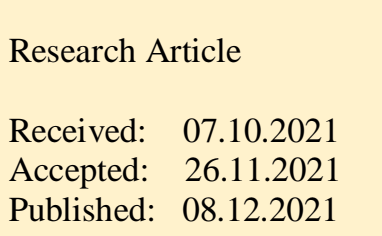 & $\begin{array}{l}\text { proposed proposals can be implemented identified by literature research and the solutions proposed for them } \\
\text { are presented to the teachers who have the most information on this topic and are at } \\
\text { the center of the problem, by means of a five-point Likert questionnaire on their } \\
\text { opinions. The identified problems and proposed solutions have also proven to be } \\
\text { correct through literature research and survey research. }\end{array}$ \\
\hline
\end{tabular}

\footnotetext{
${ }^{1}$ Çukurova Universitesi, Sosyal Bilimler Enstitüsü, Alman Dili Eğitimi Anabilim Dalı, necmettinyaman80@ outlook.com,
} 


\section{EINLEITUNG}

Das Erlernen von Sprachen ist bekanntlich ein Prozess, der intensive Zeit und viel Anstrengung erfordert. In Anbetracht dieser Situation wurde der Fremdsprachenunterricht an öffentlichen Schulen bis auf die Stufe der 2. Klassen der Grundschule abgesetzt. In den öffentlichen Schulen der Türkei wird überwiegend Englisch als erste Fremdsprache unterrichtet. Die zweite Fremdsprache wird seit dem akademischen Schuljahr 1997-1998 im Rahmen der Integrationsgesetze der Europäischen Union ab 6. Klassen als Wahlfach unterrichtet. (MEB, 1998, S. 1013). In den anatolischen und naturwissenschaftlichen Gymnasien ist die zweite Fremdsprache seit 2004-2005 Pflicht (Çelikkaya, 2013, S. 3). Im Laufe der langen Jahre wurden sich über den effektiven Sprachunterricht in den Schulen viele Gedanken gemacht, zu diesem Thema wurden viele neuen Studien durchgeführt, und das gewünschte Niveau des Fremdsprachenlernens wurde trotzdem nicht erreicht. Die unternommenen Schritte waren sicherlich wertvoll und notwendig, aber die vernünftige Zeitplanung, die Motivation und richtige Lehrmethoden wurden vernachlässigt. Das Erlernen von Fremdsprachen erfordert die Motivation, geeignete Lernatmosphäre, effektive Lerntechniken und vor allem aber die plausible Zeitplanung, ansonsten werden alle Anstrengungen und Kosten häufig unnötig verschwendet.

\subsection{Die Forschungsmethode}

Es ist notwendig, diese Forschung als theoretisch und praktisch in zwei Teile zu unterteilen. Um die aktuellen Probleme im Fremdsprachenunterricht an öffentlichen Schulen $\mathrm{zu}$ identifizieren und Lösungen vorzuschlagen, wurde eine weitreichende Literaturrecherche durchgeführt. Diese Probleme und die unter Umständen möglichen Lösungsvorschläge wurden den Lehrenden, die die meisten Informationen zu diesem Thema haben und die sich im Zentrum des Problems befinden, mittels eines Fünf-PunkteLikert-Fragebogens präsentiert. Diese Untersuchung wird als eine qualitative Forschungsmethode genannt. Bei der qualitativen Forschungsmethode wird versucht, ein bestimmtes Forschungsproblem oder thema aus der Perspektive der lokalen Bevölkerung zu verstehen. Die qualitative Forschung 2ist besonders effektiv, um kulturspezifische Informationen über Werte, Meinungen, Verhaltensweisen und soziale Kontexte bestimmter Bevölkerungsgruppen zu erhalten (Mack, Woodsong, M. Macqueen, Guest, \& Namey, 2005, S. 1).

Die durch Literaturrecherche zusammengezogenen Aussagen im Fragebogen wurden von einem Experten bei der Abteilung Messung und Bewertung in MEB kontrolliert und nach der Korrektur der technischen Fehler wurden diese Aussagen hinsichtlich der Angemessenheit der Sprache und des Zwecks von 3 Deutsch-, 3 Englisch- und 3 Türkischlehrern außerhalb der tatsächlichen Umfrageumgebung geprüft. Unzweckmäßige Aussagen wurden vom Fragebogen entfernt und die als angemessen erachteten neuen Aussagen wurden zusätzlich hinzugefügt. Die durch die verwendete Sprache verursachten Fehler wurden korrigiert. Die endgültige Fassung des Fragebogens wurde einem erziehungswissenschaftlichen Experten bei der Fakultät für Erziehungswissenschaften der Universität Mersin vorgelegt. Entsprechend seinen Empfehlungen wurde die Sprachform aller verwendeten Elemente in der Umfrage in einen ähnlichen Ausdruck umgewandelt. Nach den abschließenden Kontrollen wurde der Fragebogen in die Praxis umgesetzt.

\subsection{Ziel der Arbeit}

Ziel dieser Forschung ist es, einen effektiveren Fremdsprachenunterricht an öffentlichen Schulen zu ermöglichen. Es wird erwartet, dass Fremdsprachenlernen in den öffentlichen Schulen produktiver wird, wenn die vorgesehenen Vorschläge umgesetzt werden können. Es wird argumentiert, dass die 12 Jahre lang beim Fremdsprachenlernen verschwendeten Kosten, durch effektivere Methoden ersetzt werden können. 


\subsection{Begrenzungen}

Diese Forschung beschränkt sich auf die Antworten der Lehrer auf die Umfrage, die in den Distrikten Yenişehir und Mezitli in Mersin durchgeführt wurde. Von den an der Umfrage teilnehmenden Lehrern wird erwartet, dass sie angeben, ob sie die vom Forscher identifizierten Probleme und seine Lösungsvorschläge für diese Probleme unterstützen.

\subsection{Lehrerprofil}

Der Fragebogen wurde an 211 Deutsch- und Englischlehrer im Primar- und Sekundarbereich der staatlichen Schulen in den Bezirken, "Yenişehir und Mezitli,, von Mersin gerichtet. In der Auswertungsphase wurden bei der Prüfung der Ergebnisse der Fragebögen dank der Fragen, die die Aufmerksamkeit messen, 6 zufällig beantwortete Fragebögen von der Bewertung ausgeschlossen.

\section{BEFUNDE UND AUSWERTUNG}

Wir können die Gründe, die das Erlernen einer Fremdsprache erschweren, nicht einem einzigen Faktor in Verbindung bringen. Alle, die für das Sprachenlernen erforderlichen Elemente sollten überprüft und die Probleme bei diesen Elementen gelöst werden. Ein Fehler in einem dieser Elemente kann sich auf andere Elemente auswirken und das Erlernen der Sprache negativ beeinflussen. Die das Erlernen der Fremdsprachen erschwerenden Ursachen und Vorschläge sind wie folgt gezeigt.

\subsection{Unzureichende Anzahl der Fremdsprachenunterrichtsstunde}

Die Anzahl der Fremdsprachenkurse, die in elf Jahren angeboten werden, ist im Total nicht wenig. Diese Stunden werden jedoch auf elf Jahre verteilt, so haben einige Klassen zwei Stunden pro Woche und einige vier Stunden. Die Lernenden konzentrieren sich nicht nur auf diesen Unterricht. Sie müssen auch für alle anderen Fächer Zeit und Aufmerksamkeit geben. Die Dauer des Kontakts mit der Sprache ist gering und in der Sprache ist ständige Aufmerksamkeit erforderlich. Wenn die Lernenden die zwölfte Klasse erreichen, können sie nicht das gewünschte Niveau des Fremdsprachenlernens erreichen. Die überwiegende Mehrheit der Lernenden erreicht sogar nicht einmal das Grundniveau. Das wurde mit der Studie zwischen Britisch Council und TEPAV mit der Erlaubnis von MEB belegt und als einen Bericht veröffentlicht. (Özen, Alpaslan, \& Çăğl1, 2013, S. 9,18)

Um festzustellen, ob die Lehrenden in staatlichen Schulen den Mangel an Vorbereitungsklasse als ein Problem betrachten, wurde das den Lehrenden als eine Aussage präsentiert. Und sie haben ihre Meinungen, in dem sie eine Auswahl von Fünf-Punkte-Likert-Fragebogens treffen, erklärt. Die den Lehrenden in staatlichen Schulen präsentierte Aussage ist folgend detailliert tabellarisch erklärt.

Tabelle 1. (Prozentanteil der Aussage 1 über die Vorbereitungsklasse (Vk))

\begin{tabular}{|c|c|c|c|}
\hline \multicolumn{2}{|c|}{$\begin{array}{l}\text { Der Fremdsprachenunterricht an öffentlicher } \\
\text { Schulen ohne Vk reicht nicht für das } \\
\text { Fremdsprachenlernen aus }\end{array}$} & \multirow{2}{*}{$\frac{\text { Häufigkeit }}{2}$} & \multirow{2}{*}{$\frac{\text { Prozent }}{1,0}$} \\
\hline \multirow[t]{6}{*}{ Gültig } & Stimme gar nicht zu & & \\
\hline & Stimme nicht zu & 17 & 8,3 \\
\hline & Unentschieden & 5 & 2,4 \\
\hline & Stimme zu & 62 & 30,2 \\
\hline & Stimme voll zu & 119 & 58,0 \\
\hline & Gesamt & 205 & 100,0 \\
\hline
\end{tabular}

Der Prozentanteil für Unterstützung dieser Ansicht betrug 88,2\%. Die Unterstützungsquoten zeigen, dass die überwiegende Mehrheit der Fremdsprachenlehrer den Mangel an Vorbereitungskursen in den Schulen als ein großes Problem für das Erlernen von Fremdsprachen sieht, und dass sie die Vorbereitungsklasse als eine Notwendigkeit für Fremdsprachlernen sehen.

Auch Yalçın Dilekli präsentierte die Pilotanwendung des Englischvorbereitungskursprogramms in den fünften Klassen der Grundschule den Ansichten von 17 Lehrenden, die an 3 Pilotschulen arbeiteten. Die Lehrenden sind der Meinung, dass die Lernenden bereit sind, die Sprache in einem frühen Alter zu lernen, und dass das frühe Fremdsprachlernen 


\section{MESOPOTAMIA: JOURNAL OF INTERDISCIPLINARY STUDIES}

Volume: 1 - Issue: 1, December 2021

Necmettin YAMAN

dauerhaft sein wird und dass eine langfristige Exposition zu einem dauerhafteren Lernen führen wird. (Dilekli, 2018, S. 1400-1425)

Professor Dr. Belma Haznedar, vom Institut für Fremdsprachenunterricht der Boğaziçi Universität, betonte in ihrer Rede die Kontinuität des Fremdsprachenunterrichts und sagte, dass das Erlernen von Sprachen Kontinuität erfordere. Sobald es nicht in kurzer Zeit realisiert werde und nicht genügend Input vorliege, würden die Erfassungen in kurzer Zeit vergessen und es komme zu einem Sprachverlust. Der Spracherwerb sollte kontinuierlich sein. Die Ausbildungszeit sollte in den Folgejahren nicht verkürzt werden. Laut der Studie dauert es 5 bis 6 Jahre, bis eine Fremdsprache kommunikativ und akademisch verwendet wird. Nach einem intensiven Englischprogramm in der 5. Klasse sollte der Sprachunterricht daher in den anderen Jahren zu einem angemessenen Zeitpunkt fortgesetzt werden. Es sollte nicht auf ein Jahr begrenzt sein. (Haznedar, 2016)

In Bezug auf dieses Thema schlug der Forscher vor, die Vorbereitungsklasse sowohl auf der Primar- als auch auf der Sekundarstufe anzubieten, und stellte Lehrenden an öffentlichen Schulen direkt Fragen, um festzustellen, ob sie dieses Angebot unterstützen:

Den Befragten wurde diese Frage gestellt:

Tabelle 2. (Prozentanteil der Aussage über die Vorbereitungsklassennotwendigkeit)

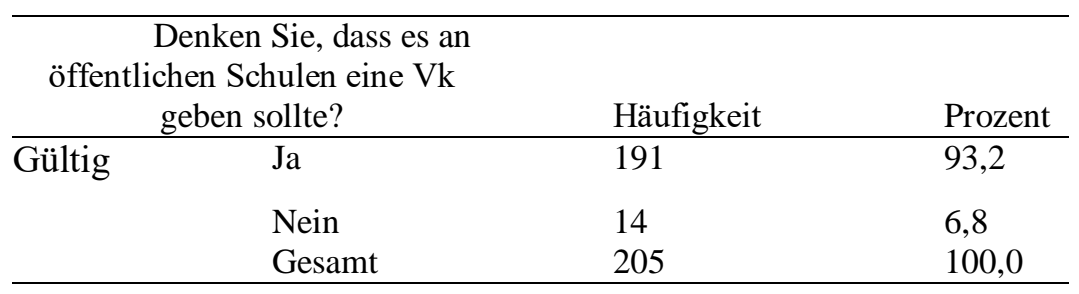

93,2 \% der Befragten beantworteten diese Frage mit Ja und nur 6,8 \% fanden die Vorbereitungsklasse als unnötig.

Die zweite Frage wurde den Befragten, die für die Notwendigkeit der Vorbereitungsklasse ausgesprochen haben, gestellt:

„Auf welchem Schulniveau sollte Ihrer Meinung nach die Vorbereitungsklasse sein?“

Die Antwort besteht aus drei Auswahlmöglichkeiten:

1. Auf Grundschulstufe 2. Auf Sekundarschulstufe 3. Auf beiden Schulstufen

Tabelle 3. (Prozentanteil der Frage über den Vorbereitungsklassenort)

\begin{tabular}{llcc}
\hline \multicolumn{2}{c}{ Auf welchem Schulniveau sollte Ihrer } & & \\
& Meinung nach die Vk sein? & & \\
& & Häufigkeit & Prozent \\
\hline Gültig & Auf Grundschulstufe & 48 & 23,4 \\
& Auf Sekundarschulstufe & 49 & 23,9 \\
& Auf beiden Schulstufen & 94 & 45,9 \\
& Gesamt & 191 & 93,2 \\
Fehlend & System & 14 & 6,8 \\
Gesamt & & 205 & 100,0 \\
& & & \\
\hline
\end{tabular}

Während die höchste Unterstützungsquote mit $45,9 \%$ für beide Schulstufen betrug, befürworteten 23,4\% der Lehrenden auf die Grundschulstufe und 23,9\% auf die Sekundarschulstufe.

All diese Ergebnisse unterstützten die Verteidigung des Forschers für die Notwendigkeit einer Vorbereitungsklasse für das Erlernen von Fremdsprachen und dass es sehr vorteilhaft wäre, diese Vorbereitungsklasse in beiden Schulstufen einzubeziehen. 


\subsection{Mangeln an Motivation}

Motivation ist ein sehr allgemeines Konzept und der sensibelste Teil des Prozesses. Tatsächlich wird es von allen anderen Elementen des Prozesses beeinflusst und hat den wichtigsten Anteil am Fremdsprachenlernen. Die Hauptgründe für die mangelnde Motivation sind in drei Punkte unterteilt. Die Lehrerzentrierten Unterrichtveranstaltungen, die nutzlosen Lernmaterialien und die mangelnde Anwendungsumgebung. Die Motivation hängt auch davon ab, ob alle anderen Elemente problemlos oder mit minimalen Problemen funktionieren. Die Motivation für die Fremdsprache zu schaffen ist am wichtigsten. Um Motivation zu erhöhen, könnten einige Maßnahmen ergriffen werden. Wie zum Beispiel:

a. Die schülerzentriert bearbeiteten Fremdsprachenkursen an öffentlichen Schulen erhöht die Lernmotivation.

b. Die Entwicklung von Fremdsprachenunterrichtsmaterialien an öffentlichen Schulen, um verschiedene Sinne anzusprechen, wird die Motivation zum Sprachenlernen erhöhen.

c. Die Bereitstellung einer fremdsprachigen Anwendungsumgebung durch das effektive Nutzen von Internetmöglichkeiten, erhöht die Lernmotivation der Lernenden.

Nach Arslan und Akbarov sollten die Lernenden in der Türkei mit technologiebasierten Sprachlehrmethoden Fremdsprachenlernen gefördert werden, weil technologische Lerninstrumente das Erlernen von Fremdsprachen erleichtert haben. Das Fernsehen, das wichtigste technologische Kommunikationsmittel, ist aus dem täglichen Leben nicht mehr wegzudenken. TV und Radio Sendungen im Fremdsprachen sind so nah wie möglich, wenn Sie nur einen Knopfdruck drücken. Selbst das Befolgen diese technologischen Mittel kann Menschen dazu motivieren, eine Sprache zu lernen. (Arslan \& Akbarov, 2010, S. 189)

$\mathrm{Ob}$ auch die Lehrenden in den staatlichen Schulen mit diesen Vorschlägen zur Erhöhung der Motivation einverstanden sind, wurden sie durch den Fragebogen den Meinungen der Lehrenden gestellt. Und die Ergebnisse sind folgend detailliert aufgelistet.

Tabelle 4 : (Prozentanteil des Vorschlags 1 über die Motivation)

\begin{tabular}{clll}
\hline \multicolumn{2}{c}{$\begin{array}{c}\text { Der schülerzentrierte Fremdsprachenunterricht an } \\
\text { öffentlichen Schulen erhöht die Lernmotivation }\end{array}$} & Häufigkeit & Prozent \\
\hline Gültig & Stimme nicht zu & 6 & 2,9 \\
& Unentschieden & 11 & 5,4 \\
& Stimme zu & 80 & 39,0 \\
& Stimme voll zu & 108 & 52,7 \\
Gesamt & 205 & 100,0 \\
\hline
\end{tabular}

Mit 91,7\% Anzahl hat diese Ansicht eine große Unterstützung bekommen. Während 5,4 \% der Befragten hier unentschieden blieben, war die Anzahl der Nicht zustimmten nur 2,9\%.

Tabelle 5 : (Prozentanteil der Aussage 2 über die Motivation)

Die Entwicklung von Fremdsprachenunterrichtsmaterialien an öffentlichen Schulen, die verschiedene Sinne ansprechen, kann

\begin{tabular}{clcc} 
die Motivation zum Sprachenlernen steigern & Häufigkeit & Prozent \\
\hline Gültig & stimme gar nicht zu & 2 & 1,0 \\
& unentschieden & 6 & 2,9 \\
& stimme zu & 51 & 24,9 \\
stimme voll zu & 146 & 71,2 \\
& Gesamt & 205 & 100,0 \\
\hline
\end{tabular}

$\mathrm{Zu}$ dieser Ansicht des Forschers gaben fast alle Befragten Unterstützungen. Die Unterstützungsrate betrug 96,1\%. 
Tabelle 6 : Prozentanteil der Aussage 3 über die Motivation)

\begin{tabular}{|c|c|c|c|}
\hline \multicolumn{3}{|c|}{ Die Zurverfügungstellung einer fremdsprachigen } & Prozent \\
\hline \multirow{6}{*}{ Gültig } & Stimme gar nicht $\mathrm{zu}$ & 2 & 1,0 \\
\hline & Stimme nicht $\mathrm{zu}$ & 2 & 1,0 \\
\hline & Unentschieden & 13 & 6,3 \\
\hline & Stimme zu & 63 & 30,7 \\
\hline & Stimme voll zu & 125 & 61,0 \\
\hline & Gesamt & 205 & 100,0 \\
\hline
\end{tabular}

Informations- und Kommunikationstechnologien (IKT)

Die Unterstützungsquote dieser Ansicht war mit 91,7 \% sehr hoch.

\subsection{Probleme bei den Unterrichtstechniken beim Fremdsprachlernen}

Grammatik-Übersetzungsmethode ist in den öffentlichen Schulen der Türkei weit verbreitet. Die Verwendung dieser Methode beim Unterrichten von Sprachen, die mit dem Ziel entwickelt wurde, tote Sprachen zu unterrichten führt in unserem Land und in der Welt zu unvollständigem Lernen bei den lebendigen Fremdsprachen. In Bezug auf unser grammatikalisches Prüfungssystem kann der Erfolg dieses Lehrsystems erwähnt werden. Es ist jedoch die Tatsache, dass die Schüler, die mit dieser Lehrmethode aufwachsen, die erlernten Sprachen nicht in die Praxis umsetzen können.

Beim Fremdsprachenlernen sollten vier Grundfertigkeiten in Betracht gezogen werden. Das sind Leseverstehen, Hörverstehen, Schreiben und Sprechen. Leider werden in meisten Schulen, besonders in staatlichen Schulen, meist nur Leseverständnis und Grammatikunterricht vermittelt. Diese Fähigkeiten sind möglicherweise sinnvoll für diejenigen, die ein akademisches Studium absolvieren möchten, aber für diejenigen, die mit dieser Sprache kommunizieren möchten reichen diese nicht aus.

Ausgehend von der Lehrerausbildung sollten die Methoden und Techniken angewendet werden, die sich auf die Kommunikation basieren und vier Fähigkeiten entwickeln. Es sollten Umgebungen geschaffen werden, in denen die Schüler an dem Kurs aktiv teilnehmen können, in denen sie nicht passiv sind und sie sollten nicht nur Informationen lernen, sondern auch die erlernten Informationen nutzen können. Die heutige Technologie ist dafür sehr gut geeignet. Es ist ein großer Vorteil, Kinder, die den Computer immer dabei haben, zu ermutigen, diese Technologie zum Erlernen von Sprachen zu verwenden.

Um herauszufinden, ob die Lehrer in den öffentlichen Schulen mit diesen Problemen und Vorschlagen einverstanden sind, wurden diese durch den Fragebogen ihren Meinungen präsentiert. Die Ergebnisse sind folgend detailliert aufgelistet.

Tabelle 7 : (Prozentanteil der Aussage 1 über die Lehrtechniken)

\begin{tabular}{llcc}
\hline $\begin{array}{c}\text { Der auf } G \ddot{U} M \text { basierende Fremdsprachenunterricht reicht } \\
\text { nicht aus, eine Fremdsprache zu lernen }\end{array}$ & Häufigkeit & Prozent \\
\hline Gültig & stimme gar nicht zu & 5 & 2,4 \\
& stimme nicht zu & 9 & 4,4 \\
& unentschieden & 16 & 7,8 \\
& stimme zu & 91 & 44,4 \\
stimme voll zu & 84 & 41,0 \\
Gesamt & 205 & 100,0 \\
\hline
\end{tabular}

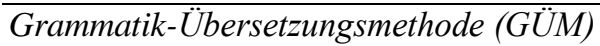

Mit der Ansicht des Forschers waren die meisten Lehrenden einverstanden. Die Unterstützungsquote der Lehrenden in den staatlichen Schulen war 85,4\%. 
Tabelle 8 : (Prozentanteil der Aussage 2 über die Lehrtechniken)

\begin{tabular}{cccc}
\hline $\begin{array}{c}\text { Der unzureichende Einsatz der Sprechfertigkeit an } \\
\text { öffentlichen Schulen macht den } \\
\text { Fremdsprachenunterricht mangelhaft }\end{array}$ & Häufigkeit & Prozent \\
\hline Gültig & Stimme gar nicht zu & 2 & 1,0 \\
& Stimme nicht zu & 3 & 1,5 \\
& Unentschieden & 2 & 1,0 \\
& Stimme zu & 70 & 34,1 \\
Stimme voll zu & 128 & 62,4 \\
Gesamt & 205 & 100,0 \\
\hline
\end{tabular}

$\mathrm{Zu}$ dieser Ansicht des Forschers haben fast alle befragten Unterstützungen gegeben. Die Unterstützungsrate betrug 96,5\%. Weil die Unterstützungsrate so hoch betrug, ähnelt sich die Rate auch bei den Lehrerprofilen.

Tabelle 9 : (Prozentanteil der Aussage 3 über die Lehrtechniken)

\begin{tabular}{|c|c|c|c|}
\hline \multicolumn{4}{|c|}{$\begin{array}{c}\text { Der unzureichende Einsatz der Hörfertigkeit an } \\
\text { öffentlichen Schulen macht den } \\
\text { Fremdsprachenunterricht mangelhaft }\end{array}$} \\
\hline \multirow[t]{6}{*}{ Gültig } & Stimme gar nicht zu & 1 & 5 \\
\hline & Stimme nicht zu & 3 & 1,5 \\
\hline & Unentschieden & 3 & 1,5 \\
\hline & Stimme zu & 72 & 35,1 \\
\hline & Stimme voll zu & 126 & 61,5 \\
\hline & Gesamt & 205 & 100,0 \\
\hline
\end{tabular}

Wie bei der Sprechfertigkeit ist die überwiegende Mehrheit der Lehrer der Ansicht, dass auch bei den Hörfertigkeiten in den Schulen mangelhaft seien. Die Rate, die davon überzeugt sind, betrug $96,6 \%$.

Tabelle 10 : (Prozentanteil der Aussage 4 über die Lehrtechniken)

\begin{tabular}{llcc}
\hline \multicolumn{2}{l}{$\begin{array}{l}\text { Durch das Ausbalancieren der vier Grundfertigkeiten (Hören, } \\
\text { Sprechen, Lesen und Schreiben) im Fremdsprachenunterricht } \\
\text { wird das Fremdsprachenlernen effektiver }\end{array}$} & Häufigkeit & Prozent \\
\hline Gültig & Stimme nicht zu & 2 & 1,0 \\
& Unentschieden & 5 & 2,4 \\
& Stimme zu & 43 & 21,0 \\
& Stimme voll zu & 155 & 75,6 \\
\cline { 2 - 4 } & Gesamt & 205 & 100,0 \\
\hline
\end{tabular}

Dem Vorschlag des Forschers, der behauptet, dass durch Ausbalancieren der vier Fertigkeiten das Fremdsprachlernen effektiver wird, gaben die überwiegende Mehrheit der Lehrer Unterstützung. Die Unterstützungsrate betrug 96,6 \%.

Tabelle 11 : (Prozentanteil der Aussage 5 über die Lehrtechniken)

\begin{tabular}{cccc}
\hline $\begin{array}{c}\text { Durch den Einsatz schülerzentrierter Methoden und } \\
\text { Techniken wird das Erlernen von Fremdsprachen effektiver }\end{array}$ & Häufigkeit & Prozent \\
\hline Gültig & Stimme gar nicht zu & 2 & 1,0 \\
& Stimme nicht zu & 3 & 1,5 \\
& Unentschieden & 9 & 4,4 \\
Stimme zu & 67 & 32,7 \\
& Stimme voll zu & 124 & 60,5 \\
Gesamt & 205 & 100,0 \\
\hline
\end{tabular}

Die Befragten gaben diesem Vorschlag mit 93,2 \% Unterstützung. 


\subsection{Probleme bei der Lehrerausbildung}

Nach Aussagen von İsmail Yaman sinkt die Bereitschaft der Studenten erheblich, die an diesen Fremdsprachprogrammen teilnehmen, insbesondere aufgrund der zunehmenden Zahl von Universitäten und Universitätsprogrammen im ganzen Land. Und es ist nicht zu leugnen, dass einige Studenten ihren Abschluss machen und ernannt werden, bevor sie ihre Englischkenntnisse auf ein ausreichendes Niveau bringen können. Aus diesem Grund empfiehlt İsmail, dass die Dozenten die Abschlussphase in ernst nehmen müssen, und dass sie den Studenten, die erforderlichen Verdienste nicht erbringen können, nicht lassen, zum Abschluss zu kommen. Je mehr die Anzahl der unfähige Fremdsprachenlehrer steigt, desto mehr fällt Fremdsprachenunterricht Debatte unweigerlich in einen Teufelskreis in der Türkei. (Yaman, 2018, S. 167)

$\mathrm{Ob}$ es in der Lehrerausbildung oder im normalen Fremdsprachenunterricht, gehören die Methoden und Techniken des Fremdsprachenunterrichts, sowie die Bewertungsmethode der Schüler zu den wichtigsten Problemfaktoren beim Sprachenlernen. Die Tatsache, dass die ausgewählten Kandidaten nicht über die erforderlichen Qualifikationen verfügen, die mangelnde schülerzentrierte und kommunikationsorientierte Ausbildung und die Tatsache, dass Absolventen ernannt werden können, ohne die erforderlichen Kompetenzen zu erreichen, führt dazu, dass das Problem beim Erlernen von Fremdsprachen in einen Teufelskreis gerät.

Demirpolat hat für die Probleme im Zusammenhang mit vorberuflicher Ausbildung der Fremdsprachenlehrer folgende Lösungsvorschläge gegeben:

- Nach Demirpolat sollte für die Fremdsprachenbereiche an Universitäten bestimmte Qualitätsstandards eingeführt werden.

- um Fremdsprachenlehrer auszubilden, sollten in Hochschuleinrichtungen ausbildete Akademiker mit Muttersprachlern angestellt werden.

- Es sollten verschiedene Projekte entwickelt werden, in denen Kandidaten für Fremdsprachenlehrer vor dem Dienst im Zielland mindestens ein Jahr studieren können. (Demirpolat, 2015, S. 16)

Studierende, die für die Fremdsprachenbereiche ausgewählt werden, sollten nicht nur durch theoretischen Teil der Universitätsprüfung ausgewählt werden, sondern auch durch ihre praktischen Fähigkeiten wie Sprech- und Hörbereichen, und es sollte sichergestellt werden, dass während ihrer Ausbildung vier Fähigkeiten entwickelt werden. Weil die Lehrer die Schüler genauso erziehen, wie sie ausgebildet werden.

$\mathrm{Ob}$ die lehrerbezogenen Probleme in den Schulen geben und ob die Lehrenden mit den Vorschlägen des Vorschers einverstanden sind, wurde durch den Fragebogen festgestellt.

Tabelle12: (Prozentanteil der Aussage 1 über die Rolle der Lehrenden)

\begin{tabular}{|c|c|c|c|}
\hline \multicolumn{2}{|c|}{$\begin{array}{c}\text { Die unzureichende Hörverstehfertigkeit bestimmter } \\
\text { Fremdsprachenlehrenden führt dazu, dass die Lernenden sich } \\
\text { diese Fertigkeit nicht aneignen }\end{array}$} & Häufigkeit & Prozent \\
\hline \multirow[t]{6}{*}{ Gültig } & Stimme gar nicht zu & 1 &, 5 \\
\hline & Stimme nicht zu & 28 & 13,7 \\
\hline & Unentschieden & 34 & 16,6 \\
\hline & Stimme zu & 89 & 43,4 \\
\hline & Stimme voll zu & 53 & 25,9 \\
\hline & Gesamt & 205 & 100,0 \\
\hline
\end{tabular}

Fast $70 \%$ der Lehrenden unterstützten diese Ansicht des Forschers. Eine beachtliche Quote von $16 \%$ blieb unentschieden und 14\% der Lehrer widersprachen dieser Ansicht. 
Tabelle 13 : (Prozentanteil der Aussage 2 über die Rolle der Lehrenden)

\begin{tabular}{cccc}
\hline \multicolumn{2}{c}{$\begin{array}{c}\text { Die unzureichende Sprechfertigkeit bestimmter } \\
\text { Fremdsprachenlehrenden führt dazu, dass die }\end{array}$} & \\
\multicolumn{2}{c}{ Lernenden sich diese Fertigkeit nicht aneignen } & Häufigkeit & Prozent \\
\hline Gültig & Stimme gar nicht zu & 1 & 5 \\
& Stimme nicht zu & 20 & 9,8 \\
& Unentschieden & 26 & 12,7 \\
& Stimme zu & 93 & 45,4 \\
Stimme voll zu & 65 & 31,7 \\
Gesamt & 205 & 100,0 \\
\hline
\end{tabular}

Diese Feststellung des Forschers wurde von einer hohen Quote von 77,1\% unterstützt. Während 12,7\% unentschieden waren, stimmten 9,8\% dieser Feststellung nicht zu.

Tabelle 14 : (Prozentanteil der Aussage 3 über die Rolle der Lehrenden)

\begin{tabular}{clcc}
\hline \multicolumn{2}{c}{$\begin{array}{c}\text { Die Beseitigung der Mängel bei Lehrenden durch } \\
\text { Fortbildung im Dienst würde einen großen Beitrag } \\
\text { zum Fremdsprachenlernen und -lehren leisten }\end{array}$} & Häufigkeit & Prozent \\
\hline Gültig & Stimme gar nicht zu & 7 & 3,4 \\
& Stimme nicht zu & 10 & 4,9 \\
& Unentschieden & 26 & 12,7 \\
& Stimme zu & 85 & 41,5 \\
Stimme voll zu & 77 & 37,6 \\
Gesamt & 205 & 100,0 \\
\hline
\end{tabular}

Während die überwiegende Mehrheit mit 79,1\% der Teilnehmer diese Aussicht des Forschers unterstützte, blieb eine bemerkenswerte Gruppe von 12\% unentschieden. Die Rate derjenigen, die nicht einverstanden waren, betrug $8,3 \%$.

\subsection{Ineffektive Unterrichtsstunden}

Einer der Faktoren, die den Erfolg des Fremdsprachenlernens steigern, ist die Nutzung der verfügbaren Zeit und Möglichkeiten. Aufgrund des Prüfungssystems in der Türkei geben die Schüler mehr Bedeutung zu einigen Unterrichten im Vergleich zu Anderen. Der Schüler lernt hart an den Fächern, die als Fragen in der Prüfung für Universität erscheinen, und misst den Fächern, die in der Prüfung Fragen nicht erscheinen, keine erforderliche Bedeutung bei. Dieser Fall wird sich viel mehr gefühlt, besonders wenn sich die Prüfungen nähern.

Auf dem Symposium des British Council und an der ITU stellten Experten zu dem Thema Fremdsprachenlernen einige Probleme und Empfehlungen vor. Einer der Faktoren, die die Englischkenntnisse erheblich beeinflussten, war die mangelnde Bedeutung des Fremdsprachenlernens wegen der Vorbereitung auf Aufnahmeprüfung der Universität. Als Lösung wurde vorgeschlagen, fremdsprachige Fragen in der Aufnahmeprüfung der Universität zu stellen. (Ülker, 2017, S. 22)

Da sich die Schüler der 12. Klasse intensiv auf Universitätsprüfungen vorbereiten müssen, werden die in diesen Klassen angebotenen Fremdsprachenunterrichte ineffizient. Daher gibt es zwei Möglichkeiten, diese Unterrichte nützlicher zu machen. Eine davon ist, in Prüfungen Fragen aus Fremdsprachen zu stellen. Die Andere ist ineffiziente Unterrichte im aktuellen System in die unteren Klassen einzuteilen. Dann kann man mehr von diesen Unterrichten profitieren.

Tabelle 15 : (Prozentanteil der Aussage 1 über die oberen Klassen)

\begin{tabular}{|c|c|c|c|}
\hline \multicolumn{4}{|c|}{$\begin{array}{l}\text { Die Tatsache, dass die Lernenden der 12. Klasse wegen der } \\
\text { Vorbereitung auf die Universitätsprüfung die Fremdsprache } \\
\text { vernachlässigen, macht das Erlernen der Fremdsprache } \\
\text { unzureichend }\end{array}$} \\
\hline \multirow[t]{5}{*}{ Gültig } & Stimme gar nicht $\mathrm{zu}$ & 4 & 2,0 \\
\hline & Stimme nicht $\mathrm{zu}$ & 21 & 10,2 \\
\hline & Unentschieden & 18 & 8,8 \\
\hline & Stimme zu & 78 & 38,0 \\
\hline & Stimme voll zu & 84 & 41,0 \\
\hline
\end{tabular}


Tabelle 15 : (Prozentanteil der Aussage 1 über die oberen Klassen)

\begin{tabular}{|c|c|c|c|}
\hline \multicolumn{2}{|c|}{$\begin{array}{l}\text { Die Tatsache, dass die Lernenden der 12. Klasse wegen der } \\
\text { Vorbereitung auf die Universitätsprüfung die Fremdsprache } \\
\text { vernachlässigen, macht das Erlernen der Fremdsprache } \\
\text { unzureichend }\end{array}$} & Häufigkeit & Prozent \\
\hline \multirow[t]{6}{*}{ Gültig } & Stimme gar nicht zu & 4 & 2,0 \\
\hline & Stimme nicht $\mathrm{zu}$ & 21 & 10,2 \\
\hline & Unentschieden & 18 & 8,8 \\
\hline & Stimme zu & 78 & 38,0 \\
\hline & Stimme voll zu & 84 & 41,0 \\
\hline & Gesamt & 205 & 100,0 \\
\hline
\end{tabular}

Die Unterstützungsquote für diese Problemfeststellung des Forschers war hoch. Während die Unterstützungsquote 79\% betrug, waren die Anteile der Gegner und Unentschiedenen insgesamt 21\%.

Tabelle 16 : (Prozentanteil der Aussage 2 über die oberen Klassen)

Die Aufnahmeprüfung muss fremdsprachige Fragen enthalten,

von denen alle Teilnehmer verantwortlich sein müssen. Das würde dazu führen, dass auch die Lernenden oberer Klassen die

\begin{tabular}{clcc} 
& Fremdsprache(n) ernst nehmen & Häufigkeit & Prozent \\
\hline \multirow{6}{*}{ Gültig } & Stimme gar nicht zu & 5 & 2,4 \\
& Stimme nicht zu & 20 & 9,8 \\
& Unentschieden & 22 & 10,7 \\
& Stimme zu & 65 & 31,7 \\
& Stimme voll zu & 93 & 45,4 \\
& Gesamt & 205 & 100,0 \\
\hline
\end{tabular}

Der Vorschlag des Forschers, dass die Schüler in den zwölften Klassen mehr Wert auf das Sprachlernen legen, wenn sie in den Aufnahmeprüfungen auch mit den Fragen von den Fremdsprachen ausgesetzt werden, bekam eine große Unterstützung von Lehrenden in den staatlichen Schulen. Die Unterstützungsquote betrug $77,1 \%$. Die Rate der unentschiedenen und nicht einverstandenen ist jedoch nicht zu unterschätzen.

Tabelle 17 : (Prozentanteil der Aussage 3 über die oberen Klassen)

\begin{tabular}{llcc}
\hline $\begin{array}{c}\text { Die Verlegung des Fremdsprachenunterrichts der 12. Klasse } \\
\text { in untere Klassenstufen würde das Fremdsprachenlernen } \\
\text { effektiver machen }\end{array}$ & Häufigkeit & Prozent \\
\hline Gültig & Stimme gar nicht zu & 15 & 7,3 \\
& Stimme nicht zu & 31 & 15,1 \\
& Unentschieden & 51 & 24,9 \\
& Stimme zu & 47 & 22,9 \\
& Stimme voll zu & 61 & 29,8 \\
& Gesamt & 205 & 100,0 \\
\hline
\end{tabular}

Der zweite Alternativvorschlag des Forschers war die Verlegung der nicht ausreichend genutzten Unterrichte in den Zwölften Klassen in die unteren Klassen. Das bekam die wenigste Unterstützungsquote unter allen Vorschlägen. Die Unterstützungsquote betrug 52,7 \%. Bei diesem Vorschlag blieben viele Lehrer unentschieden. Die Rate der Unentschiedenen war 24,9\%. Und 22,4\% stimmten diesem Vorschlag nicht zu. Der größte Unterschied bei diesem Vorschlag wurde auf Fachbereichsbasis festgestellt.

Tabelle 18 : (Fachbereichsprofil der Lehrenden für die Aussage 3)

\begin{tabular}{llcc}
\hline \multirow{2}{*}{$\begin{array}{c}\text { Die Verlegung des Fremdsprachenunterrichts der 12. Klasse } \\
\text { in untere Klassenstufen würde das Fremdsprachenlernen } \\
\text { effektiver machen }\end{array}$} & \multicolumn{2}{c}{ Fachbereich } \\
\cline { 2 - 4 } & Anzahl & Deutsch & Englisch \\
\hline Stimme gar nicht zu & \% nach Fachbereich & $3,0 \%$ & 14 \\
\multirow{2}{*}{ Stimme nicht zu } & Anzahl & 0 & $8,1 \%$ \\
& \% nach Fachbereich &, $0 \%$ & 31 \\
Unentschieden & Anzahl & 4 & $18,0 \%$ \\
& \% nach Fachbereich & $12,1 \%$ & $27,3 \%$
\end{tabular}


MESOPOTAMIA: JOURNAL OF INTERDISCIPLINARY STUDIES

Volume: 1 - Issue: 1, December 2021

Necmettin YAMAN

\begin{tabular}{llcc} 
Stimme zu & Anzahl & 11 & 36 \\
& \% nach Fachbereich & $33,3 \%$ & $20,9 \%$ \\
Stimme voll zu & Anzahl & 17 & 44 \\
& \% nach Fachbereich & $51,5 \%$ & $25,6 \%$ \\
Gesamt & Anzahl & 33 & 172 \\
& \% nach Fachbereich & $100,0 \%$ & $100,0 \%$ \\
\hline
\end{tabular}

Das auffälligste Ergebnis des Fragebogens war bei dieser Aussage und besonders bei der Unterstützungsquote nach dem Profil der Lehrenden. Während die Unterstützungsquote der Deutschlehrenden bei $84,8 \%$ betrug, das heißt die überwiegende Anteil der Deutschlehrenden; blieb der Anteil der Englischlehrenden unter 50\%, dh 46,5\%. Eine große Anzahl der Englischlehrenden, also $27,3 \%$, blieben bei diesem Vorschlag unentschieden. Es wird geschätzt, dass ein wichtiger Faktor daran ist, dass Deutschunterricht nur an Gymnasien und mit wenigen Unterrichtszahlen gegeben wird. Deutschlehrenden wollen nicht, dass ihr ohnehin schon weniger Unterricht verschwendet wird.

\section{SCHLUSSFOLGERUNGEN UND VORSCHLÄGE}

Die Schlussfolgerungen nach der Umfrage sind wie folgt:

- Die überwiegende Mehrheit der Fremdsprachenlehrenden findet den Mangel an Vorbereitungsklasse an den Schulen als ein großes Problem für das Erlernen der Fremdsprachen. Diese Ergebnisse unterstützen die These über die Notwendigkeit der Vorbereitungsklasse und ihre Einführung auf beiden Schulstufen.

- Die hohen Unterstützungsquoten der Lehrenden zeigen, dass der Einsatz von schülerzentrierten Lehrmethoden, der geeigneten Unterrichtmaterialien und der effektiven Nutzung der Möglichkeiten des Internets die Motivation zum Lernen einer Fremdsprache erhöhen wird.

- Die dritte Hypothese war, dass im Fremdsprachenunterricht in Schulen überwiegend Grammatik erteilt wird und dass Sprechen und Hören vernachlässigt werden. Als Lösung für diese Probleme wird der Einsatz schülerzentrierter und kommunikationsorientierter Methoden und Techniken befürwortet, die die Ausbalancierung aller Fertigkeiten ermöglichen vorgeschlagen haben. Die hohen Unterstützungsquoten der Lehrenden bewiesen diese Feststellungen und die Notwendigkeit dieser Lösungsvorschläge.

- Die Feststellungen über die Mangelhaften bei bestimmten Lehrkräften wurden auch durch die Umfrage bewiesen. Als Lösung wurde die Fortbildung im Dienst vorgeschlagen. Der Lösungsvorschlag, dass die Beseitigung der Mängel bei diesen Lehrenden durch Fortbildungen im Dienst zum Sprachenlernen beitragen würde, hat eine große Unterstützung gefunden.

- Es hat sich ergeben, dass die Lernenden der 12. Klasse sich auf Grund der Aufnahmeprüfung nicht auf den Fremdsprachenunterricht konzentrieren können. Die Unterstützungsquote der Lehrenden in Sekundarschulen ist höher als die der Lehrenden in anderen Schulen, weil sie dieses Problem genauer beobachten können. Diese Arbeit hat auch ergeben, dass die interuniversitäre Aufnahmeprüfung Fragen aus der ersten und zweiten Fremdsprache enthalten sollte, und dass der Fremdsprachenunterricht der 12. Klasse in untere Klassenstufen verlegt werden sollte. Der andere Lösungsvorschlag über die Verlegung der Fremdsprachenunterrichtsstunden der 12. Klassen in die unteren Klassenstufen bekam 
weniger Unterstützung als alle anderen Vorschläge. Die Unterstützer dieses Vorschlags sind meistens Deutschlehrende. Das war das auffälligste Ergebnis der Umfrage, weil die Lehrenden beider Fächer in dieser Sicht größtenteils anderer Meinung waren. Das hat wohl damit zu tun, dass Deutsch nur an Gymnasien und zwar mit wenigen Stunden unterrichtet wird. Und die Deutschlehrenden wollen nicht, dass ihre ohnehin wenigen Unterrichtsstunden verschwendet werden. Trotzdem hat auch dieser Ansicht bei der Mehrheit der Lehrenden Zustimmung gefunden.

\section{BIBLIOGRAPHIE}

Arslan, M., \& Akbarov, A. (2010). Türkiyede Yabancı Dil Öğretiminde Motivasyon-Yöntem Sorunu ve Çözüm Önerileri. Selçuk Üniversites iEdebiyat Fakültesi Dergisi, 189.

Çelikkaya, Y. Ş. (2013). Ortaöğretim Öğrencilerinin İkinci Yabancı Dil Almanca. Middle Eastern \& African Journal of Educational Research, 5, (3).

Demirpolat, B. C. (Temmuz 2015). Türkiyenin Yabancı Dil Öğretimiyle İmtihanı Sorunlar ve Çözüm Önerileri. Analiz 131, (16).

Dilekli, Y. (2018). Ortaokul İngilizce Hazırlık Sınıfı Programı Pilot Uygulamasının Öğretmen Görüşlerine Göre Değerlendirilmesi. Opus, 1401-1411.

Dilekli, Y. (2018). Ortaokul İngilizce Hazırlık Sınıfı Programı Pilot uygulamasının öğretmen görüşlerine göre değerlendirilmesi. Opus, 1400-1425.

Haznedar, B. (2016). 5. Sınıfta İngilizce Hazırlık Programı'nın önemi BAU'da düzenlenen panelde tartıştık; http://www.bahcesehir.k12.tr/tr/kurumsal/haberlerdetay/5-Sinifta-Ingilizce-HazirlikPrograminin-onemi-BAUda-duzenlenenpanelde-tartistik/260/2295/0 (Abgerufen am 29. 10. 2019)

Mack, N., Woodsong, C., M. Macqueen, K., Guest, G., \& Namey, E. (2005). Qualitative Research Methods. North Carolina: Family Health International.

MEB. (1998). İlköğretim Okulu Seçmeli Dersler Listesi. MEB Tebliğler Dergisi, 1013.

Özen, E. N., Alpaslan, İ. B., \& Çağlı, A. (2013). Türkiye'deki Devlet Okullarında. Ankara: Mattek Matbaacılık Basım Yayın Tanıtım Ticaret Sanayi LTD. ŞTİ.

Ülker, N. (2017). Yabancı Dil Eğitimi Sorunlar ve Çözüm Önerileri. İtü Vakfi Dergisi, 22.

Yaman, İ. (2018). Türkiye'de İngilizce Öğrenmek: Zorluklar ve Firsatlar. Rumelide Dil ve Edebiyat Araştırmaları Dergisi, 167.

\section{EXTENDED ABSTRACT}

As it is well known, language learning is a process that requires intensive time and effort. In view of this situation, foreign language teaching in public schools has been discontinued up to the 2nd grade of primary school. English is the primary foreign language taught in Turkish public schools. The second foreign language has been taught as an elective since the academic school year 1997-1998 within the framework of the integration laws of the European Union from 6th grade. The second foreign language has been compulsory in Anatolian and science high schools since 2004-2005. Although students in public schools in Turkey received hundreds of hours of foreign language lessons for 11 years, most of them realised at the end of school that they could hardly speak a word of these 
foreign languages. There are many causes of which lack of motivation, aimlessness, incorrect teaching methods, need for well-trained teachers, and unreasonable limited hours seem to be the most important. The aim of this research is to enable more effective foreign language teaching in public schools. Foreign language learning in public schools is expected to be more productive if the proposals can be implemented. It is argued that the 12 years of wasted money and efforts for the learning a foreign language can be replaced by more effective methods. The problems of learning foreign languages in public schools identified by literature research and the solutions proposed for them are presented to the teachers who have the most informations on this topic and are at the center of the problem, by means of a five-point Likert questionnaire on their opinions. The identified problems and proposed solutions have also proven to be correct through literature research and survey research.

The conclusions of the survey are as follows: 1 . The majority of foreign language teachers find the lack of preparatory classes in schools to be a major problem for language learning. These results support the thesis about the necessity of the preparatory class and its introduction to both levels of the school. 2. The high support rates of the teachers shows that the use of student-centered teaching methods, the appropriate teaching materials and the effective use of the possibilities of the Internet will increase the motivation to learn a foreign language. 3 . The third hypothesis was that grammar is predominantly taught in foreign language teaching in schools and that speaking and listening are neglected. As a solution to these problem, the use of student-centered and communication-oriented methods and techniques is advocated, which have proposed a balancing of all skills. The high support rates of the teachers proved these findings and the necessity of these proposed solutions. 4. The findings about deficiencies in certain teachers were also confirmed by the survey. On-the-job training was suggested as a solution. The proposed solution to eliminate the shortcomings of these teachers through on-the-job training in language learning has met with strong support. 5. It turned out that the 12th grade Students cannot concentrate on foreign language lessons because of the entrance exam. The support rate of teachers in highschools is higher than that of teachers in primary schools because they can observe this problem more closely. This work has also shown that the inter-university entrance examination should contain questions from the first and second foreign language, and that foreign language teaching in the 12th grade should be moved to lower grades. The other proposed solution of moving foreign language lessons from the 12th grade to the lower grades received less support than all the other suggestions. The supporters of this proposal are mostly German teachers. That was the most striking result of the survey because the teachers of both subjects largely disagreed on this point of view. That probably has to do with the fact that German is only taught at highschools with a few hours. And the German teachers don't want their already few lessons to be wasted. Nevertheless, this Suggestion also met with approval from the majority of teachers. 